\title{
Cardiac arrest in pregnancy: Case report and review of the literature
}

\author{
S Budhram, FCOG (SA), MMed (O\&G) \\ Department of Obstetrics and Gynaecology, Faculty of Medicine and Health Sciences, Stellenbosch University, Tygerberg, Cape Town, South Africa
}

Corresponding author: S Budhram (samant@absamail.co.za)

Cardiac arrest in pregnancy is a rare event, and resuscitation of the pregnant patient is complicated by the impact on resuscitative measures of the normal physiological changes of pregnancy. A case of successful resuscitation of a pregnant patient with a cardiac arrest and a normal neurocognitive outcome is reported.

S Afr J Obstet Gynaecol 2015;21(1):10-11. DOI:10.7196/SAJOG.929

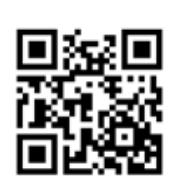

\section{Case report}

A 38-year-old woman, gravida 5 para 4, booked at 14 weeks' gestation. She had a history of ischaemic heart disease, chronic hypertension and hypercholesterolaemia, and had discontinued her medication (without medical advice) 5 months earlier because she was planning a pregnancy with her new partner. Additional risk factors identified were advanced maternal age, four preterm vaginal deliveries (the last of which was 8 years previously), a history of heavy smoking, and the current pregnancy being a twin gestation.

There was a delay in referral of the patient to the tertiary centre, and she was first seen at the combined obstetric-cardiac clinic at 22 weeks' gestation. At this visit she was noted to be in a stable condition with satisfactory vital parameters and an intact twin gestation in keeping with 22 weeks' gestational age (GA). The cardiologist proceeded to perform an echocardiogram while attempts were being made to retrieve the patient's notes relating to previous cardiology consultations.

Within 15 minutes of commencing the examination, the patient became acutely dyspnoeic. At this stage the limited echocardiogram showed left ventricular dysfunction with a left ventricular ejection fraction (LVEF) of $30 \%$. The patient was immediately commenced on oxygen therapy and transferred to the obstetric critical care unit (OCCU) in a semirecumbent, left lateral position.

On arrival in the OCCU, a rapid initial assessment revealed that the patient was in severe respiratory distress with signs suggestive of frank pulmonary oedema. She received a bolus of $80 \mathrm{mg}$ furosemide intravenously (IV). Resuscitative measures had been commenced by a multidisciplinary team (MDT) comprising an anaesthetist, an intensivist, a fellow in maternal-fetal medicine and intensive caretrained nursing staff, when the patient suffered a cardiac arrest. She was immediately intubated in a rapid-sequence fashion, and cardiopulmonary resuscitation (CPR) was undertaken with the patient maintained in a $15^{\circ}$ lateral tilt. She remained asystolic after 5 minutes of CPR and a total of $3 \mathrm{mg}$ IV-administered adrenaline. A decision was made to perform a perimortem hysterotomy to assist maternal resuscitative efforts. In the absence of operative instruments, a sterile 20 inch blade was used to perform the operation, with delivery of stillborn twins and the placenta. Within 10 seconds the patient was documented to have a return of spontaneous circulation (ROSC), after a total of 6 minutes of asystole. The uterus and abdomen were closed with the aid of a suture pack and the patient was commenced on broad-spectrum antibiotic therapy and a concentrated infusion of oxytocin. She was initially ventilated with a fraction of inspired oxygen of $100 \%$ and a positive end-expiratory pressure of $10 \mathrm{~cm} \mathrm{H}_{2} \mathrm{O}$ and maintained on infusions of dobutamine, adrenaline, furosemide, morphine and midazolam.

A review by the cardiology team, with the patient's previous cardiology notes, revealed that she had suffered a myocardial infarction (MI) 4 years ago. Investigations at that time revealed triple-vessel coronary artery disease and she was deemed not a suitable candidate for coronary artery bypass grafting or stent placement. She was to be considered for cardiac transplantation if her lifestyle could be modified.

Echocardiography, electrocardiograms and serial cardiac enzymes following this acute event were not suggestive of an acute MI. A diagnosis of cardiac failure in the background of severe ischaemic left ventricular dysfunction was made. This was probably precipitated and exacerbated by the exaggerated physiological changes accompanying the multiple gestation.

The plan to wean the patient off ventilation and the infusions and continue antifailure therapy was successfully achieved in the next 48 hours, and she was assessed to be neurocognitively intact. She was recommenced on her chronic medication, and extensively counselled about the events that had occurred since admission, the vital importance of compliance with medical therapy and lifestyle modification. She was counselled against any future pregnancies and received a long-acting reversible contraceptive. A follow-up visit with the obstetric and cardiology teams was scheduled with a view to reassessment of her suitability for surgical intervention.

\section{Discussion}

Ischaemic left ventricular dysfunction, which in this case was probably a consequence of a previous $\mathrm{MI}$, is characterised by dilatation and impaired systolic function of one or both ventricles (LVEF < 40\%). Presenting manifestations can include arrhythmias, heart failure and sudden cardiac arrest.

Cardiac arrest in pregnancy is a rare but catastrophic event, with an estimated incidence of $1 / 30000$ pregnancies. ${ }^{[1]}$ The principles 
of management include timely identification, initiation of CPR, treatment of readily correctible causes and expedited delivery of the fetus, within 4 - 5 minutes, to achieve optimal outcomes.

The normal physiological changes of pregnancy need to be considered when resuscitating the pregnant patient. These include:

- Difficult airway resulting from pharyngeal oedema and enlarged breast tissue

- Increased risk of aspiration due to relaxation of the oesophageal sphincter

- Decreased chest wall compliance secondary to enlarged breasts

- Decreased functional residual capacity due to upward displacement of the diaphragm by the gravid uterus

- Sequestration of up to $30 \%$ of the circulating blood volume in the latter half of pregnancy, due to compression of the inferior vena cava, iliac vessels and abdominal aorta by the gravid uterus

- Decreased effect of chest compressions as a result of decreased venous return, leading to supine hypotension

- Obstruction of forward flow of blood by the gravid uterus, especially in cardiac arrest, where the arterial pressure and volume are already reduced.

In general, resuscitative algorithms are the same for pregnant as for non-pregnant patients, with a few exceptions to accommodate the normal physiological changes of pregnancy. These are:

- Placing the patient in the left lateral position (at least $15^{\circ}$ tilt) or manually displacing the uterus to the left of the abdomen

- Early intubation, correctly applied cricoid pressure and use of a smaller-sized endotracheal tube

- Consideration of perimortem hysterotomy/caesarean section early in resuscitative efforts. ${ }^{[2]}$

Studies ${ }^{[3,4]}$ have shown that there are cases in which ROSC does not occur until the uterus is emptied by a perimortem hysterotomy/caesarean section. In addition, there are many case reports of unsuccessful resuscitation attempts with usual life-support measures, but which noted ROSC only after the uterus was emptied.

When deciding to perform a perimortem delivery, the following factors need to be considered:

- GA $<20$ weeks - an emergency hysterotomy is unlikely to improve the situation

- GA 20 - 23 weeks - an emergency hysterotomy may improve the likelihood of survival of the mother, although survival of the fetus is very unlikely

- GA >24 weeks - emergency caesarean section will be likely to rescue both the mother and the fetus. ${ }^{[5]}$
Importantly, delivery needs to be embarked upon within 4 - 5 minutes of cardiac arrest to achieve optimal maternal and/or fetal outcomes.

Successful resuscitation is reported in $6-15 \%$ of patients who suffer an in-hospital cardiac arrest, although survival rates are likely to be lower in pregnant patients, as a result of the physiological changes of pregnancy complicating resuscitation. A patient whose heartbeat and respiration have ceased for less than 4 minutes has an excellent chance of recovery with CPR and advanced cardiac life support. Brain damage may occur after 4 minutes and becomes certain after 6 minutes. ${ }^{[6]}$

Many leading healthcare facilities around the world employ percutaneous extracorporeal membrane oxygen (ECMO) in selected patients with cardiac-respiratory arrest, as part of their resuscitation algorithm. ECMO can provide both cardiac and respiratory support to patients whose heart or lungs are so damaged that they cannot perform their function. This is a temporising measure to allow time to address the problem that caused the arrest. There is a growing body of evidence of improved success and outcomes with the use of ECMO during resuscitation.

\section{Conclusion}

Cardiac arrest in pregnancy is a rare event. Early aggressive resuscitation by a well-trained MDT increases the chance of a successful outcome for the mother and/or fetus, as shown in our case report. Adherence to standard CPR algorithms with modifications to accommodate the physiological changes of pregnancy with early (within 4 - 5 minutes) hysterotomy/caesarean section may well benefit both the mother and the fetus.

In summary, our case illustrates that an optimal outcome for cardiac arrest in pregnancy is possible in a unit that is well set up (with trained staff and a fully equipped resuscitation area). This is only achievable with ongoing in-service training and 'fire drills' in resuscitation, as is the case in our unit.

1. Atta E, Gardner M. Cardiopulmonary resuscitation in pregnancy. Obstet Gynecol Clin N Am 2007;34(3):585-597. [http://dx.doi.org/10.1016/j.ogc.2007.06.008]

2. Soar J, Perkins GD, Abbas G, et al. European Resuscitation Council Guidelines for Resuscitation 2010 Section 8. Cardiac arrest in special circumstances: Electrolyte abnormalities, poisoning, drowning, accidental hypothermia, hyperthermia, asthma, anaphylaxis, cardiac surgery, trauma, pregnancy, electrocution. Resuscitation 2010;81(10):1400-1433. [http://dx.doi.org/10.1016/j. resuscitation.2010.08.015]

3. Katz V, Balderston K, DeFreest M. Perimortem cesarean delivery: Were our assumptions correct? Am J Obstet Gynecol 2005;192(6):1916-1920. [http://dx.doi.org/10.1016/j.ajog.2005.02.038]

4. Dijkman A, Huisman CM, Smit M, et al. Cardiac arrest in pregnancy: Increasing use of perimortem caesarean section due to emergency skills training? BJOG 2010;117(3):282-287. [http://dx.doi. org/10.1111/j.1471-0528.2009.02461.x]

5. Farinelli CK, Hameed AB. Cardiopulmonary resuscitation in pregnancy. Cardiol Clin 2012;30(3):453-461. [http://dx.doi.org/10.1016/j.ccl.2012.04.006]

6. ECC Committee, Subcommittees and Task Forces of the American Heart Association. 2010 American Heart Association Guidelines for Cardiopulmonary Resuscitation and Emergency Cardiovascular Care. Circulation 2010;122:S640-861. [http://dx.doi.org/10.1161/circulationaha.110.970889] 